\title{
Computer-based automatic finger- and speech-tracking system
}

\author{
BJÖRN BREIDEGARD \\ Lund University, Lund, Sweden
}

\begin{abstract}
This article presents the first technology ever for online registration and interactive and automatic analysis of finger movements during tactile reading (Braille and tactile pictures). Interactive software has been developed for registration (with two cameras and a microphone), MPEG-2 video compression and storage on disk or DVD as well as an interactive analysis program to aid human analysis. An automatic finger-tracking system has been implemented which also semiautomatically tracks the reading aloud speech on the syllable level. This set of tools opens the way for large scale studies of blind people reading Braille or tactile images. It has been tested in a pilot project involving congenitally blind subjects reading texts and pictures.
\end{abstract}

In the study of blind people's tactile reading there has been a demand for more efficient technology for performing large scale studies including efficient analysis and evaluation tools. Computer based technologies have been developed only very recently (Breidegard et al., in press). Earlier studies were based on video technology and manual analyses. Bertelson and Mousty designed registration equipment based on two television cameras, a microphone, a digital timer, a video combiner, and a video recorder and carried out two studies (Bertelson, Mousty, \& D'Alimonte, 1985; Mousty \& Bertelson, 1985). Data were combined and recorded on a video recorder. The purpose was to find out how reading speed is affected by hand usage (left hand only, right hand only, and both hands) and types of text (prose, statistical approximations, and scrambled words). The human analysis was carried out by playing back the recordings on a TV monitor. Millar $(1988,1997)$ performed a pioneering study, also based on video registration equipment similar to Bertelson and Mousty's. However, Millar used transparent Braille sheets and filmed the hand movements from below, yielding an extremely good output on the TV monitor. The reading behaviors were analyzed on the basis of manually coded video frames.

In this project, we used two cameras, one filming from below through transparent Braille sheets as did Millar, but now with computer based registration and analysis. A second camera, also connected to the computer, filmed from an angle above to reflect more holistic movements of the reading hands. A microphone registered the conversations and oral reading during the test sessions. The results of the pilot study (for which the technology described here was developed), along with its interdisciplinary backgrounds and procedures have been published (Breidegard et al., in press; Breidegard, Fellenius, Jönsson, \& Strömqvist, 2006). This article deals mainly with the technical aspects.

Automatic finger tracking enables computer based automatic analyses and large scale classifications with many subjects.

\section{TOP-DOWN DESIGN}

A top-down approach was adopted to methodically design the complete system in its interaction with human subjects: the hardware (in the form of supporting furniture, the cameras, a glass plate, the illumination, the semitransparent reading material, the microphone, the optic processing, the PC) and the software. The top-down method facilitates planning, understanding and handling of the system's complexity (Wirth, 1971).

\section{MATERIAL AND METHODS}

This design project centered on the blind test subjects and the human evaluators. It had three components: artifacts such as the reading table (with its cameras, glass plate, reading transparencies), computer hardware, and computer software.

The reading table was to fit the test subjects as well as possible so as not to disturb their reading. The computer software's interactive interfaces were to fit the human, not necessarily the technically oriented evaluators, as smoothly as possible. Different trade-offs were taken into consideration and evaluated with respect to functionality and implementation costs.

The reading table with its transparent glass plate, illumination, cameras and microphone was designed to be as simple as possible. Three cables (two cameras, one mi-

B. Breidegard, bjorn@certec.lth.se 


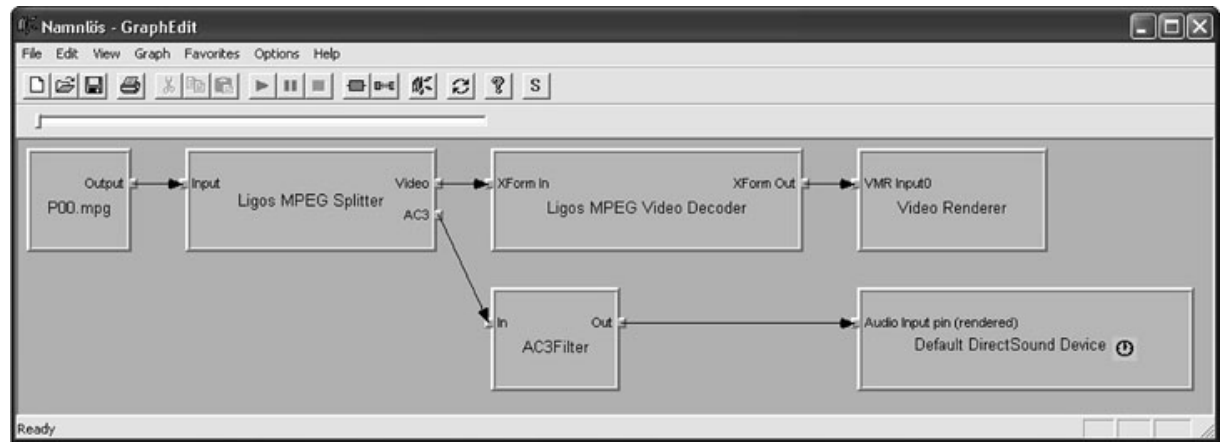

Figure 1. DirectShow filter graph rendering the video/audio file P00.mpg. The first filter, Ligos MPEG splitter, splits the file into a video stream (MPEG-2 format) and an audio stream (Dolby AC3 format). Then the two streams are decoded (decompressed) and rendered to the computer screen and loudspeakers, respectively. The graph can be built programmatically or with the GraphEdit tool. GraphEdit can also be used to rapidly test different filters and graphs before programmatically building in the final application. Finally, all graphs are generated and executed programmatically in the application program context.

crophone) were connected to a Windows XP based PC computer with inbuilt video capture hardware.

\section{Visual $\mathrm{C}++$ and DirectX}

Microsoft's Visual C ++ Version 8.0 was used for software development (Kruglinski, Shepherd, \& Wingo, 1998). The DirectX packages designed and distributed by Microsoft were extensively used in the software development. DirectShow (Linetsky, 2001; Paesce, 2003) was of great value because of the possibility to build audio/ video filter graphs either graphically (with the GraphEdit program tool) or programmatically. DirectShow highly facilitates the design of video/audio applications. With DirectShow it is easy to build applications for video recording, playback, compression/decompression and disk storage. With a special purpose filter, the sample grabber, the behavior of the filter can be programmatically defined (e.g., to perform image and audio processing). A DirectShow filter graph example is shown in Figure 1.

\section{Preexisting Software Library}

The author has used Visual $\mathrm{C}++$ for the last 8 years, and during this time has developed a large and versatile hierarchical class library including, among other things, classes for video/audio capture, playback, and digital image/audio processing. The library has been continually developed in different concurrent projects which has as a bonus enabled crossover usage between the projects.

\section{Visualizations and Interactivity}

During the whole project, in which system development was intertwined with user tests and evaluations, a strong emphasis was placed on the extensive use of visualizations and the interactive software user interface. Custom visualizations were designed for testing, development, improvement, debugging, and data presentation for the specialists in visual impairments who analyzed the results of test readings. The software user interface was designed on the same premises.

\section{SYSTEM DESIGN AND DESCRIPTION}

\section{The Small-Scale Tactile Lab}

The development of the research driven technology resulted in a small-scale tactile lab, see the schematic view in Figure 2. The main components are the reading table with its two cameras and microphone: the first camera (e) filming from below, the second (f) filming from an angle above, the microphone $(\mathrm{g})$. The other main component was the computer (h) for which suitable software was to be designed.

\section{Early Critical Decisions and Parameter Locking}

A number of early critical decisions, which would affect the outcome of the project and would be very hard to change in a later phase, had to be taken, and a number of parameters had to be locked in the early phase of the project.

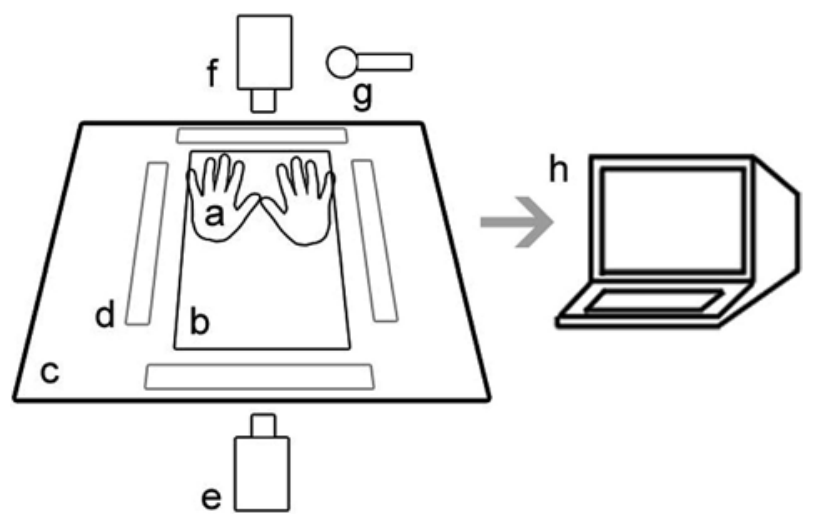

Figure 2. Schematic view of the small-scale tactile lab. (a) Reading hands. (b) Plastic semitransparent Braille page. (c) Solid, transparent glass plate also functioning as a part of the table. (d) Illumination directed upward to the Braille page. (e) Upwarddirected camera registering the fingers from below. (f) Camera registering the fingers from an angle above. (g) Microphone. (h) Computer registering and storing the two video streams. 


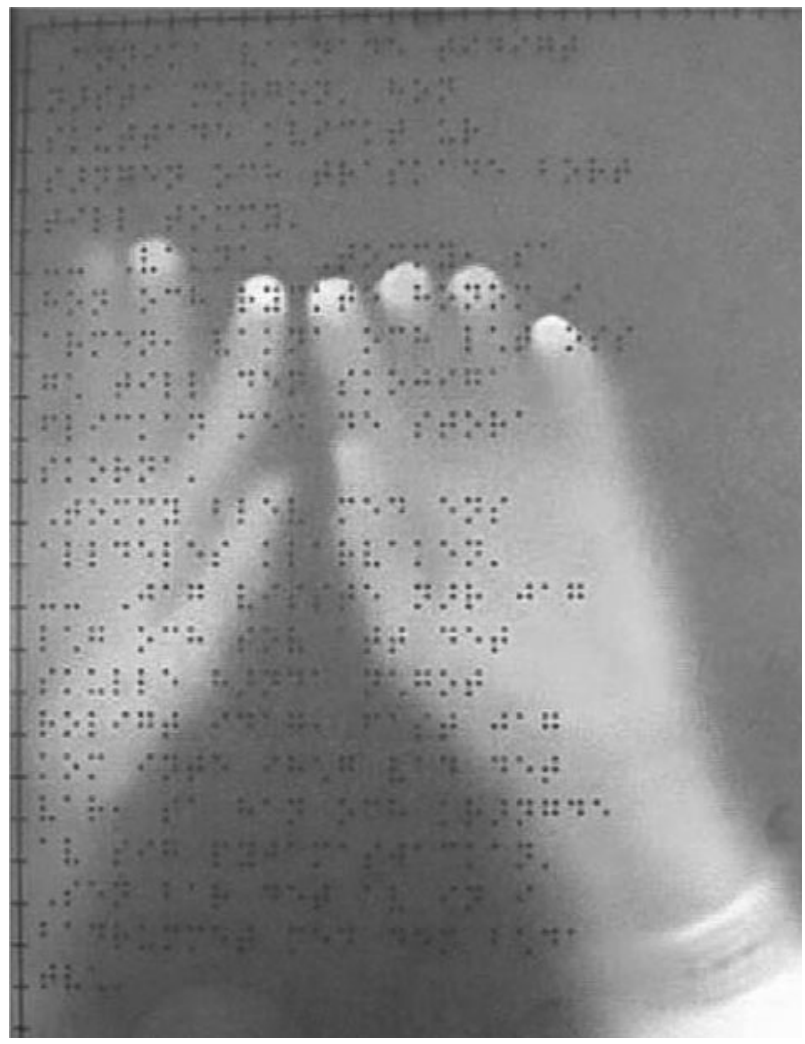

Figure 3. Camera's view of the plastic semitransparent sheet. Here, we see the Braille sheet and the reader's hands from below. The Braille dots are also printed in black to facilitate visual analysis. Calibration lines with scale marks are also printed in black. The whitening effect is clearly visible, especially for the left index finger, as an indication of pressure against the sheet. The video frames (like this image) are mirrored in the vertical axis to preserve the impression of reading direction from left to right. This gives the illusion that the hands are filmed from above, even though you are actually seeing the underside of the hands, and particularly the fingertips that carry out the actual reading.

Some of the early design considerations and decisions are the following.

- Limited size of the reading sheets. The size chosen was $26.0 \times 29.5 \mathrm{~cm}$ (former standard for regular Braille printing; the A4 format could also be used).

- Use of standard video cameras and Web cameras from an ordinary radio/TV/computer shop.

- Restricted camera resolutions and frame rates, even though large resolution and high frame rates would facilitate the automatic finger-tracking implementation. The resolution chosen for the first camera (e in Figure 2 ) was $768 \times 576$ pixels (as high as possible using a PAL standard camera), and for the second (f in Figure 2), $320 \times 240$ pixels (the resolution was reduced for this camera to save hard disk space). A frame rate of 25 frames per second (PAL standard) was chosen for the two cameras.

- The sound quality chosen was a 44.1-KHz sampling frequency, 16-bit sample resolution, and stereo.
- State-of-the-art desktop PC. A Pentium 4 (2.7 GHz, 512-MB RAM) based Windows XP PC with inbuilt video capture hardware and $300 \mathrm{~GB}$ of disk space. Today (2 years after the project start) we would use laptops, which are now in the same class.

- Critical selection of plastic material for the reading sheets. They should be moderately semitransparent to enhance the "whitening effect" of the fingertips, see Figure 3. The use of the whitening effect would facilitate the design of the finger tracking algorithms as well as the interactive analysis.

\section{THE READING TABLE}

The purpose of the reading table was to serve as an interface between the tactile reader and the registration components (cameras and microphone). The reading table was designed to be convenient for the reader, and not to disturb his or her normal way of reading. It was also designed so that the cameras and microphone could perform accurate registrations.

The physical realization can be discerned in Figure 4. Its base is a vertically adjustable table with a rectangular cut out in the wooden board. A thick glass plate covers the cut out, and the transparent Braille sheet is placed on the glass plate. The first camera is placed below the table, and the second camera on the table's farther side directed at the reader's hands. The microphone is also placed on the table.

All Braille texts and images were printed in black on plastic transparent sheets (using a specialized silk screen process) to make it possible for the camera to record the finger movements and the Braille text from below. The Braille sheets were held in place on the glass plate with plastic guides and a strip of tape.

There were some complaints about the plastic reading material used in the tests. The "feel" was different from what the subjects were used to. The transparent plastic ma-

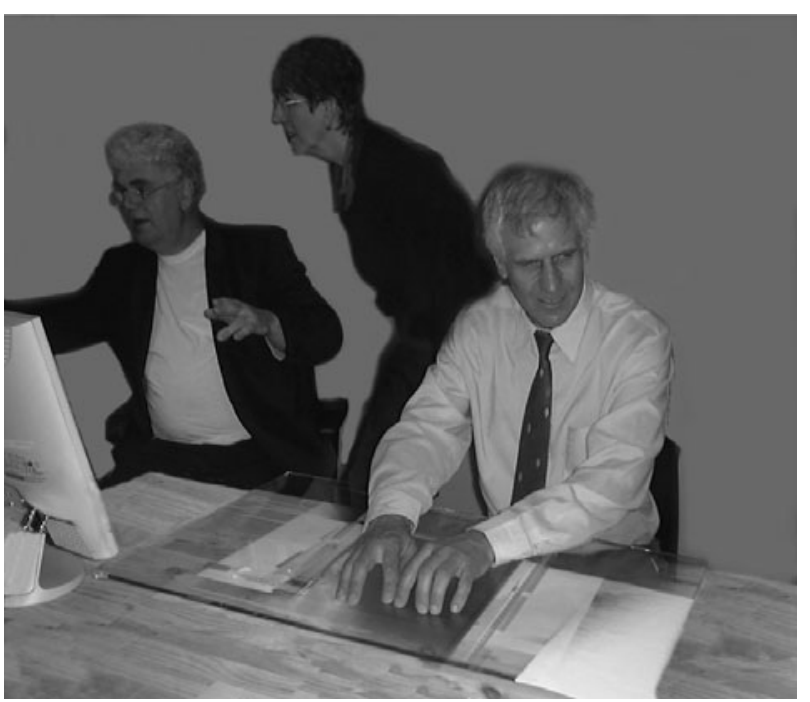

Figure 4. The reading table. S.B. (key research person, right) together with researchers in the tactile lab. 
terial used in the experiment was chosen in cooperation with experts in Braille reading. The experiment was carried out on the assumption that there would be only marginal effects on reading from the transparent material, and that the cost of presenting a slightly deviating material to the subjects would easily be counterbalanced by the significant gain of being able to capture tactile reading online. In a follow-up project, we will, among other things, compare reading rate in a condition with the transparent material to reading rate in a condition where standard Braille paper is used.

The camera registration from below produced excellent picture quality (see Figure 3). The illumination was placed under the table and directed at the semitransparent sheet from beneath. The rest of the room was in relative darkness. The whitening effect can be seen clearly as an indication of finger pressure against the sheet. Fingers not pressed against the sheet have no such effect, and the harder the fingertip is pressed, the whiter the image becomes. When the camera is filming from below the image is mirrored in the vertical axis (left becomes right and right becomes left). It is very annoying to analyze the mirrored recordings, where the readers seem to be reading from right to left. Therefore, the video from the camera is remirrored in the vertical axis (by software) before display and storage.

The cameras and microphone were directly connected to the PC with three separate cables. The camera filming from below was a standard VHS-C camera recorder (Panasonic NV-S85E, only the camera part was used) connected to the S-VHS input of the inbuilt video capture card of the PC. The camera filming from above was a Web camera (Logitech QuickCam Pro 4000) connected via USB to the PC. The microphone was connected directly to the PC's sound card. Firewire (IEEE 1394) cameras could also be used. Today we use a Pinnacle USB capture device with S-VHS and microphone inputs connected to a PC USB port. Since this is an external device, it is now possible to use a laptop computer.

\section{THE REGISTRATION PROGRAM}

The registration program serves two purposes. The first is to facilitate camera calibration and function as a camera monitor for testing the equipment; the second is to carry out the actual recording (with two cameras and one stereo microphone), data processing and storage on the hard disk. The program was designed in Visual $\mathrm{C}++$ and the audio/video manipulation was built by programmatically generated DirectShow filter graphs.

In the calibration/monitor mode, the picture from the underneath camera is shown on the computer screen. The image is mirrored in the vertical axis, cropped (to filter out the surrounding underside of the wooden table), and two red lines are shown (one horizontal and one vertical) to adjust the camera's origin and perpendicularity. These image processing operations are performed in $\mathrm{C}++$ code with the DirectShow Sample Grabber filter (see Figure 5A). The program code for the mirroring operation is shown in Figure 5B. In the Millar project (without computers) they solved the mirroring problem by using a soldering iron to reverse the wires driving the video camera's horizontal deflection coils.

In the recording mode, the two video streams are captured, mirrored, cropped, MPEG-2 compressed and finally written to the hard disk as two separate files. The sound is captured, compressed and written to the disk files. The files can be given appropriate names for each test reading. The two video files can be played back with standard media players.

The recordings were also stored on standard DVDs. This was of great benefit in that the recordings could be distributed easily among the project members and evaluated with standard DVD players, or even better with the interactive analysis program (see next section).

\section{THE INTERACTIVE ANALYSIS PROGRAM}

In the analysis phase that followed the test reading sessions, specialists in visual impairments made detailed analyses and measurements using a special purpose analysis program (see Figure 6).

\section{User Interactivity and Audio-Visual Feedback}

The program displays two video streams and one audio stream, and has basic functions such as record, play, pause, and stop. Among the added functions are playback rate selection, repetition of user selected sequences, stepping frame by frame and a manual stopwatch function. New functions were successively added to enhance the program at the researchers' requests. The program was based on programmatically generated DirectShow filter graphs, and DirectShow's application programmer's interface (API) to implement the diverse playing functions.

\section{THE AUTOMATIC FINGER-TRACKING SYSTEM}

The automatic finger tracking was divided into four steps:

1. Automatic decoding of the Braille text to plain text. The decoded plain text was stored both as a text file (containing each character in the text, its coordinates on the sheet, and also row and column numbers) and as an image file in jpg format. Most of the people involved were not able to decode Braille visually, so this step made the analyses more convenient (see Figure 7, right panel).

2. Automatic finger tracking of the two index fingers for each video frame in a recording. The two index fingers are considered to be the primary reading (decoding) fingers. Other fingers are involved in the reading process both for support, navigation and occasionally reading. Our system can be extended to track more fingers.

3. Storing the coordinates of the index fingers for each video frame in a position file that could be used by other programs or the interactive analysis program.

4. Back-annotating the interactive analysis program with the plain text image file, and the position file for each recording to be able to show the plain text and the positions of the index fingers as red and green circles (see Figure 7). 

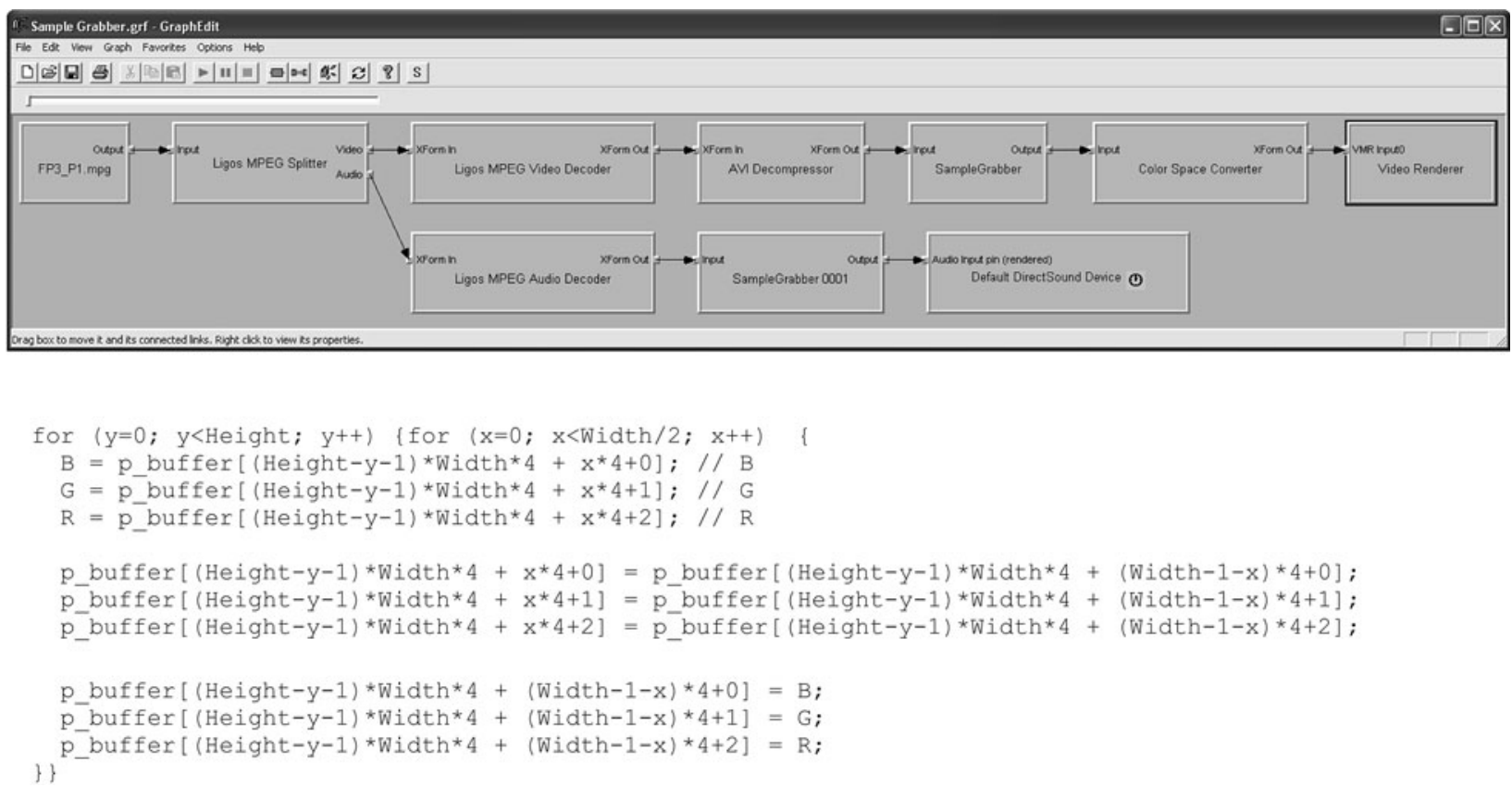

Figure 5. (A) DirectShow filter graph used for the calibration mode in the registration program. Both the video and audio paths contain sample grabbers, which can be used to access and eventually manipulate video/audio data. (B) The $\mathrm{C}++$ code executing in the video sample grabber to implement the mirroring operation.

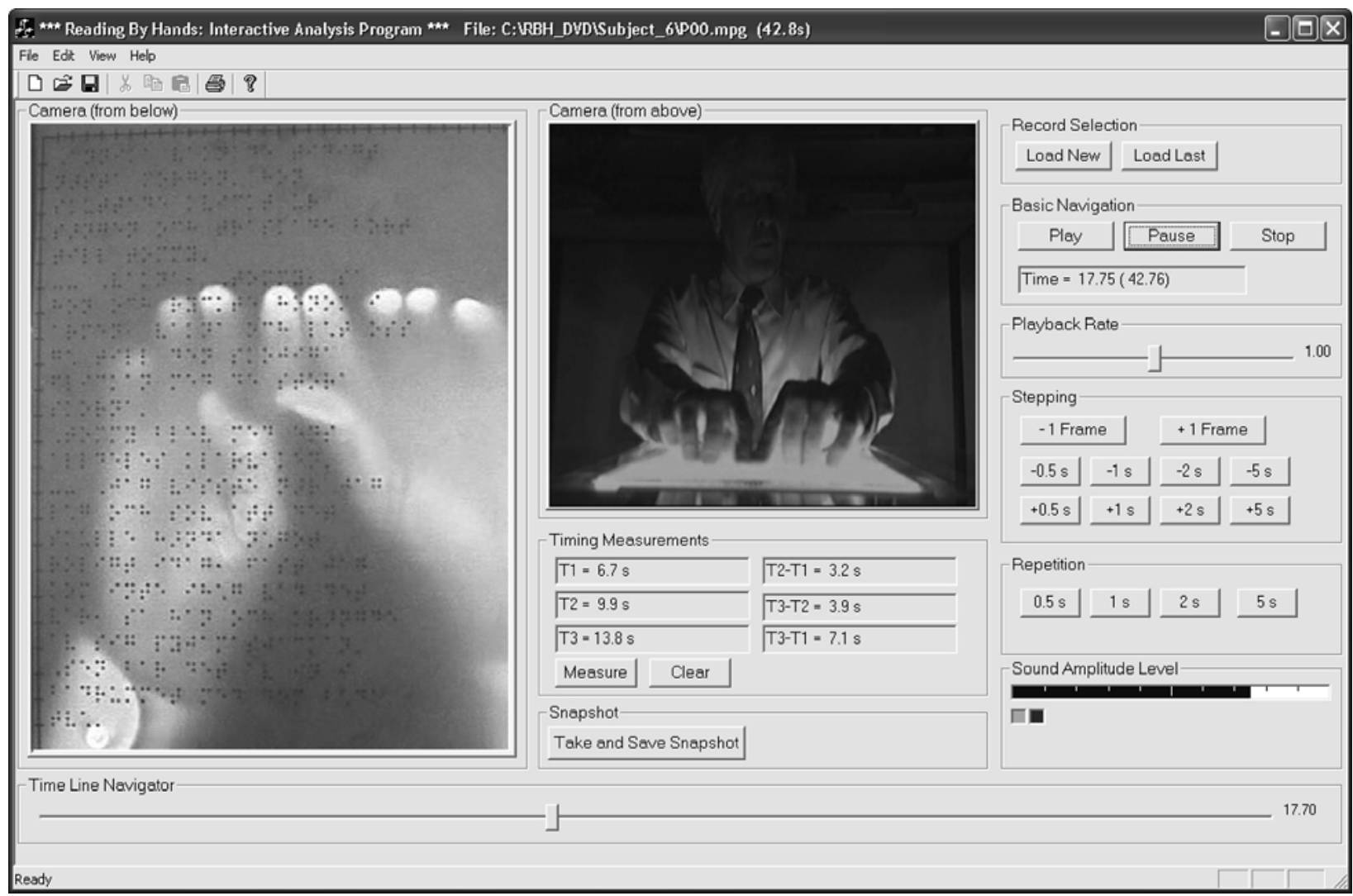

Figure 6. User interface of the interactive analysis program. The left video pane shows the image from the first camera registering from below. The whitening patches from the fingertips pressing against the Braille sheet show up distinctly. The right video pane shows the image from the second camera registering from an angle above. This view complements the view from below disclosing more about specific hand/finger constellations, finger twisting, reading rhythm, etc. The rest of the user interface controls are designed to facilitate the human analysis of the registrations such as record selection, navigation, slow motion, stepping, repetition, manual time measurements, and so on. Snapshots from the analysis can be saved and printed for documentation purposes. 

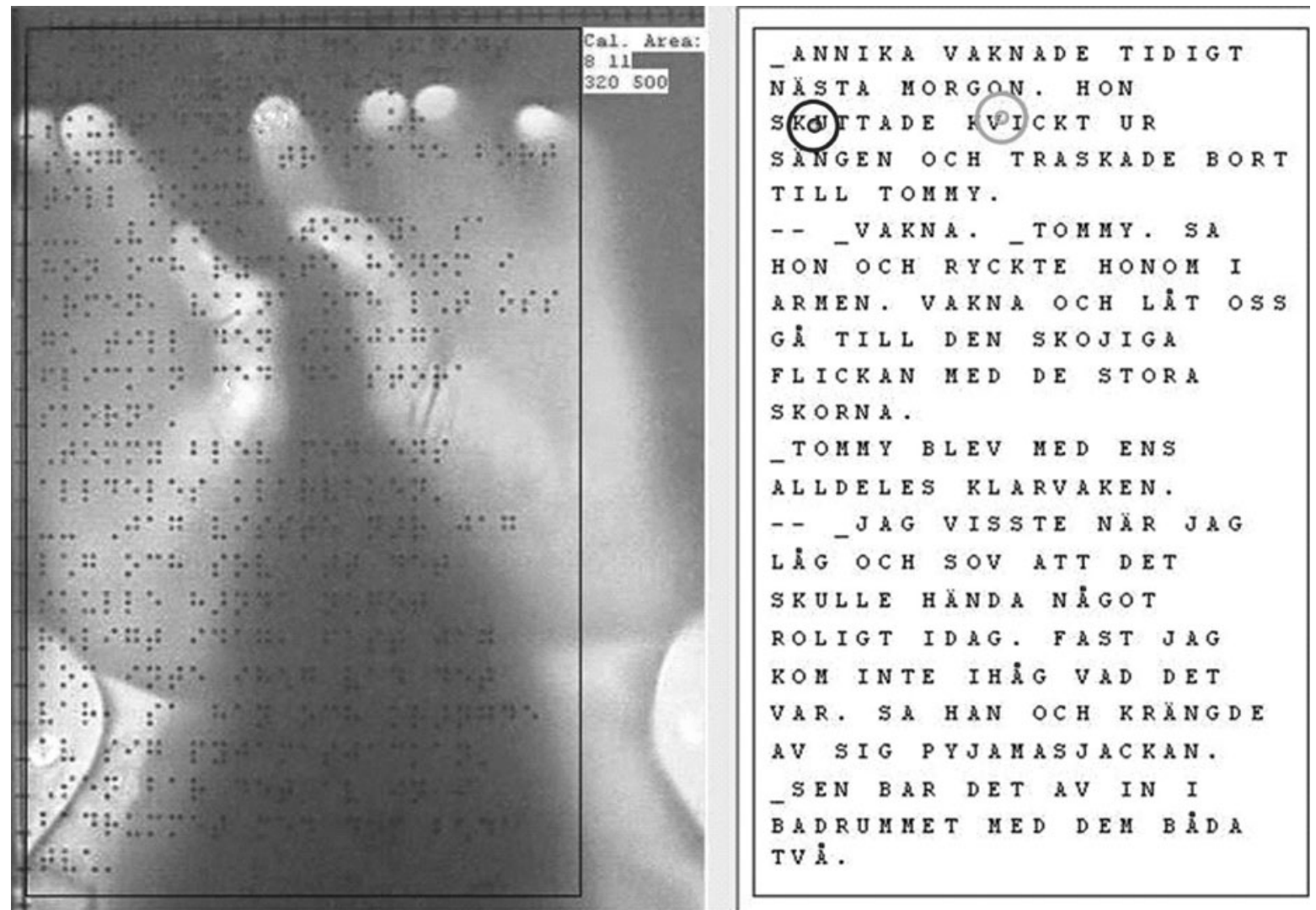

Figure 7. Interactive analysis program with back-annotated position file created by the automatic finger-tracking system. The left part shows the video recorded by the upward camera. The fingertips are naturally accentuated in that they turn white when pressed against the reading material - a property used by the automatic analysis program. The right part shows the same video image automatically analyzed with the plain text as background and the two index fingers marked by a red (left) and green (right) circle, respectively (here shown in two shades of gray).

A

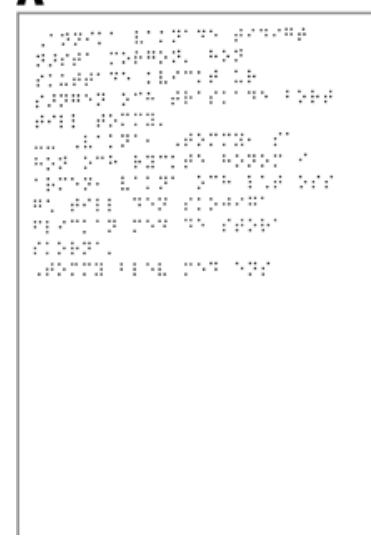

B

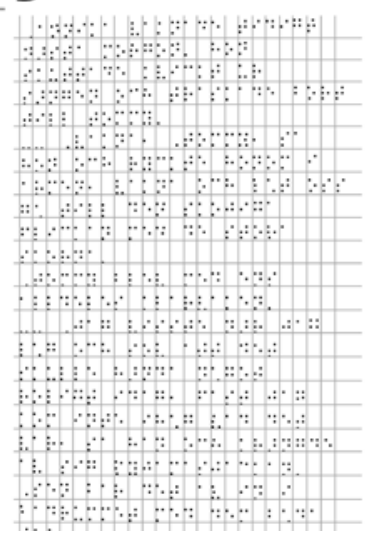

C

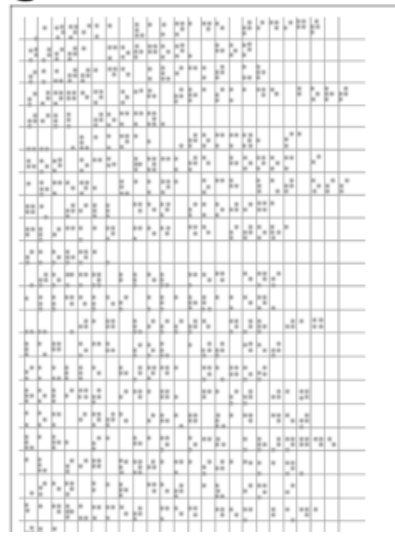

D

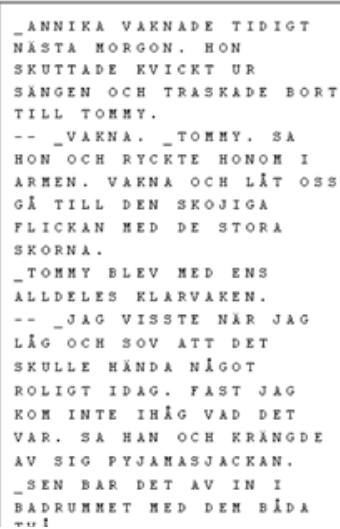

Figure 8. (A) A template matching procedure finds all Braille dots and generates this synthetic image. The computer now knows the positions for all dots. (B) Finding all Braille cells. A space analysis procedure finds all Braille cells by computing an orthogonal grid so there is exactly one Braille cell in each square. (C) An unsharpening (low-pass filtering) operation improves the following Braille cell template matching process. (D) Synthetic templates for each Braille character are swept through each grid cell. After this procedure is completed, each Braille character has been decoded into plain text. 


\section{Braille-to-Text Decoding}

Technically, the decoding from Braille to the plain text stage is based on template matching using normalized correlation:

$$
\begin{aligned}
\text { Corr } & =\frac{\hat{x}_{1} \cdot \hat{x}_{2}}{\left\|\hat{x}_{1}\right\|\left\|\hat{x}_{2}\right\|} \\
\hat{x}_{1} & =x_{1}-\text { mean }\left(x_{1}\right) \\
\hat{x}_{2} & =x_{2}-\text { mean }\left(x_{2}\right),
\end{aligned}
$$

where

\section{$\|\cdot\|$}

is the L2-norm and $x_{1}$ and $x_{2}$ are the vectors obtained by raster scanning the template bitmap and a patch of the same size as the template, respectively, in the video image.

First of all, the video frame of the Braille page is analyzed. The Braille dots are embossed and printed in black. The incoming video frame contains noise, and the illumination is not evenly distributed. The template matching algorithm finds all Braille dots and computes a new and synthetic image where all Braille dots are black, and everything else is white (see Figure 8). The next step is to identify the positions of all Braille cells (letters and digits). The white horizontal and vertical spaces between Braille cells are used to compute an orthogonal grid that guarantees that there is exactly one Braille cell somewhere in each square in the grid. This step also takes in account the spatial distortion introduced due to imperfections in the camera optics. A template for each Braille character is then swept through each grid square and the character with the highest correlation (matching score) is chosen. As an extra safety precaution, a check sum is used: the chosen Braille character must have the same number of dots as there are dots in the grid square. In this way the plain text is obtained (see Figure 7).

\section{Automatic Finger Tracking With Kalman Filter}

The automatic finger-tracking stage is based on template matching and a tracking filter algorithm called a $g-h$ filter (Brookner, 1998). Considering the one-dimensional case, and that the object (a fingertip) is moving at constant speed, the system dynamic model can be written as

$$
\begin{aligned}
& x_{n+1}=x_{n}+v_{n} \mathrm{~T} \\
& v_{n+1}=v_{n},
\end{aligned}
$$

where $v$ is the speed, $x$ the position of a fingertip (in horizontal direction), $n$ is the time step, and T the time interval between time steps. These equations constitute the base for the algorithm to estimate the new position and new speed. If the measured position $y_{n}$ of the object is not equal to the estimated position $x_{n}$, the speed is not constant-it has been changed and the estimated speed and position are predicted by the $g-h$ tracking filter equations

$$
v_{n+1}=v_{n}+h_{n} \frac{y_{n}-x_{n}}{\mathrm{~T}}
$$

and

$$
x_{n+1}=x_{n}+v_{n+1} \mathrm{~T}+g_{n}\left(y_{n}-x_{n}\right)
$$

where $g_{n}$ and $h_{n}$ are small parameters that can be time dependent. Here, constant values are used for $g_{n}$ and $h_{n}$ and related so that the tracking filter is a steady-state Kalman filter (Brookner, 1998). There are two independent $g-h$ filters: one for the horizontal direction and one for the vertical.

A synthetically generated template resembling a fingertip is used for finding the positions of fingertips. The template is circular to avoid problems with fingertips that are rotated during the reading process. The starting positions for the index fingers are marked interactively in the computer display with the mouse. The tracking algorithm automatically tracks the two finger positions and stores them on a hard disk file. The tracking algorithm based on the $g-h$ filter predicts the next two fingertip positions from current positions and speeds. The template matcher then searches a window centered on the estimated position for the best match, and assigns the best match position as the current position. The size of this search window is a compromise: as large as possible to facilitate tracking, but as small as possible to avoid confusion with other fingertips. This process continues to the end of the recording. During reading, especially of tactile images, finger constellations sometimes emerge (fingers from both hands intertwined or overlapping) that defy automatic tracking. Here, the human eye must do the analysis and recalibrate the tracking algorithm with the correct position of the fingertip. In order to include these particular events in the analyses, around ten minutes of manual calibration or trimming is needed for a 5-min recording containing 7,500 picture frames. The return sweeps during the reading of Braille text can also cause problems - they are sometimes too fast to be picked up by the current sampling rate ( 25 picture frames per second). In our further development of the AFTS, we will increase the sampling rate to minimize this type of problem.

\section{Inbuilt Visual Debugging}

The tracking algorithm was crucial, and it took much iteration to get it to work reliably. The tracking algorithm was visualized (e.g., displaying the predicted search window) and overlaid the film showing the reading fingers (left pane of Figure 7). The tracking algorithm was run frame by frame, and by watching the graphics and textual data representations of the filter variables, the tracking algorithm could be debugged, improved and trimmed to satisfaction.

\section{Visualization Results Generated by the Automatic Finger-Tracking System}

With the position file generated by the automatic fingertracking system, it was now encouraging to start analyzing the test reading data obtained. Two obvious visualizations suitable for human visual analysis are the $x-y$ diagram and the $x-t$ diagram (see Figure 9). For specialists in visual impairments, these diagrams disclose much about the test subjects' reading styles.

As seen in the diagrams, the text line reading is cyclic, and each cycle can be divided into nonoverlapping phases. We can, for example, partition the text line reading into 

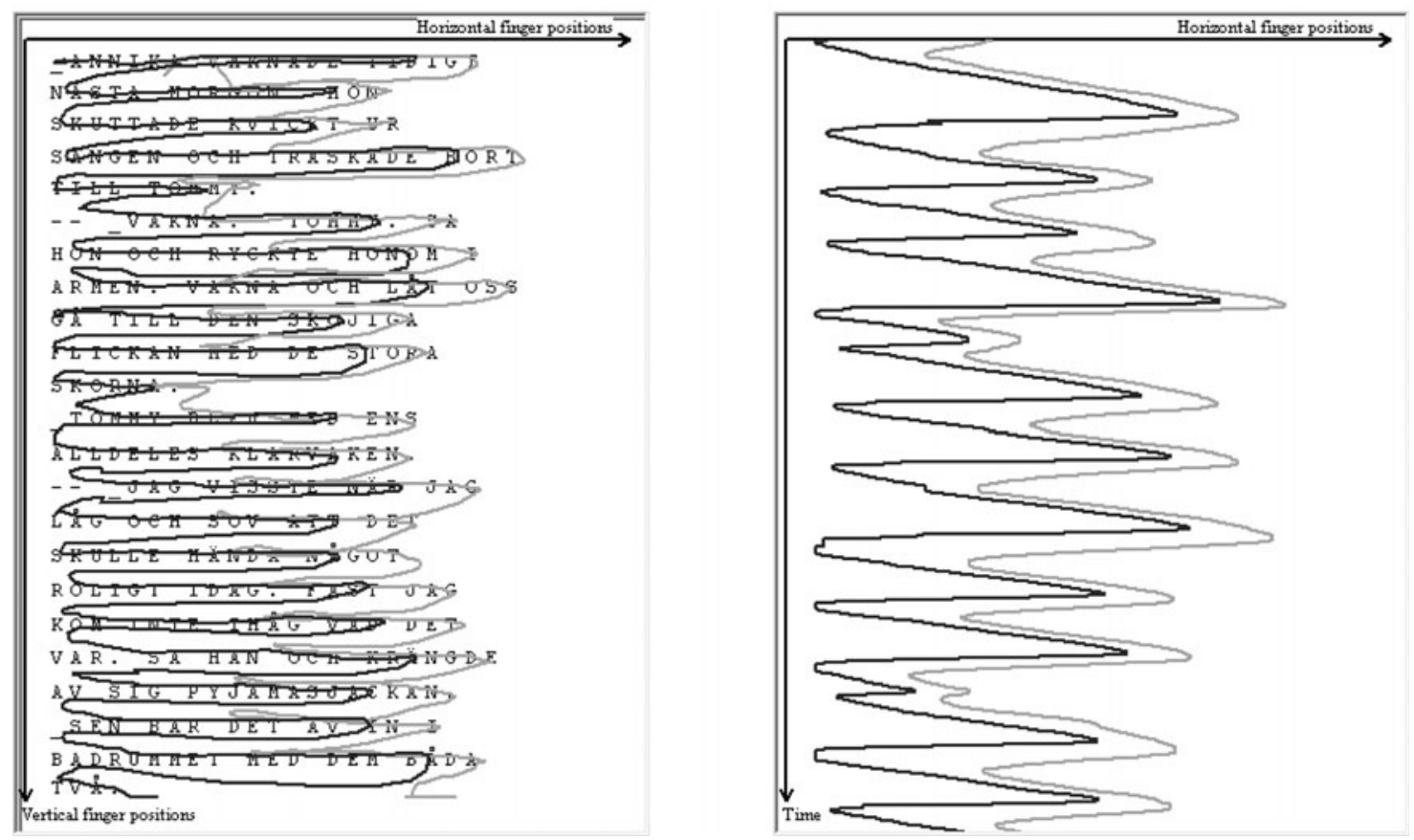

Figure 9. (A) The $x-y$ diagram shows the traces for the left (in dark gray) and right (in light gray) index fingers generated during reading of a Braille page. The Braille to plain text decoded image is shown as background to make human analysis easier. (B) The $x$-t diagram shows the two index fingers' horizontal positions on the Braille sheet on the horizontal axis, and time on the vertical axis. As can be seen in both diagrams, the test person mainly reads the left part of the sheet with the left index finger, and the right part with the right index finger. In the middle, both fingers are reading in parallel.

four phases, and let the automatic finger-tracking system compute the time percentages for each one visualized in Figure 9. The four phases chosen here are RR (both fingers are reading from left to right), LR (left finger makes a return sweep, from right to left, to the beginning of the next line, and right finger continues reading), LL (both fingers make return sweeps), and RL (left finger is reading again, and right finger is still making a return sweep). The resulting time percentages for 5 test subjects are shown in Table 1.

Subject D, for example, spends most of the time (54\%) reading with both index fingers in parallel, $24 \%$ reading with the left index finger only, and $11 \%$ with the right index finger only. Eleven percent of the time, the fingers are engaged in return sweeps, that is, phases of transportation to the next line. The left index finger is engaged in reading $78 \%$ of the total reading time and the right index

Table 1 Example From the Automatic Analysis

\begin{tabular}{lrrrrrl}
\hline & \multicolumn{4}{c}{ Percentage } & \multicolumn{1}{c}{ Hand } & \\
\cline { 2 - 5 } \multicolumn{1}{c}{ Subject } & RR & LR & LL & RL & Preference & \multicolumn{1}{c}{ Type } \\
\hline A (female) & 0 & 79 & 21 & 0 & right & one-handed \\
B (female) & 33 & 31 & 10 & 26 & right & disjointed \\
C (female) & 62 & 15 & 14 & 9 & right & mixed \\
D (male) & 54 & 11 & 11 & 24 & left & mixed \\
E (male) & 54 & 12 & 12 & 22 & left & mixed \\
\hline
\end{tabular}

Note-Phase time percentages and reading style classifications are computed automatically. finger, $65 \%$. Although S.B., our research person, is not lefthanded, his most employed reading finger was nonetheless the left index finger. AFTS also computes the reading style and hand dominance on the basis of the time percentages for the four phases. The hand preference is also anticipated based on which of the LR or RL phases dominates.

The $x-t$ diagram, shown in Figure 9B, can be extended with the computed phase time information by adding color coded horizontal stripes to further visualize the reading behaviors (see Figure 10).

\section{Tactile Picture Reading}

Hitherto, all examples have shown Braille reading. Figure 11 shows the corresponding $x-y$ diagram for a subject exploring a tactile picture of a human face. The tactile picture was printed in relief on the same type of plastic transparent sheets as the Braille texts. The picture included different relief patterns for head contour, eyes, pupils, eye brows, nose, mouth, ears, and hair. Just as in the text reading activity, the spatial exploration of the tactile image reveals that the left part is mainly decoded by the left index finger and the right part by the right index finger.

\section{THE AUTOMATIC FINGER- AND SPEECH-TRACKING SYSTEM}

After the success with automatic finger tracking and the promising automatically computed results of the tactile test readings, new ideas emerged. It would be very interesting 

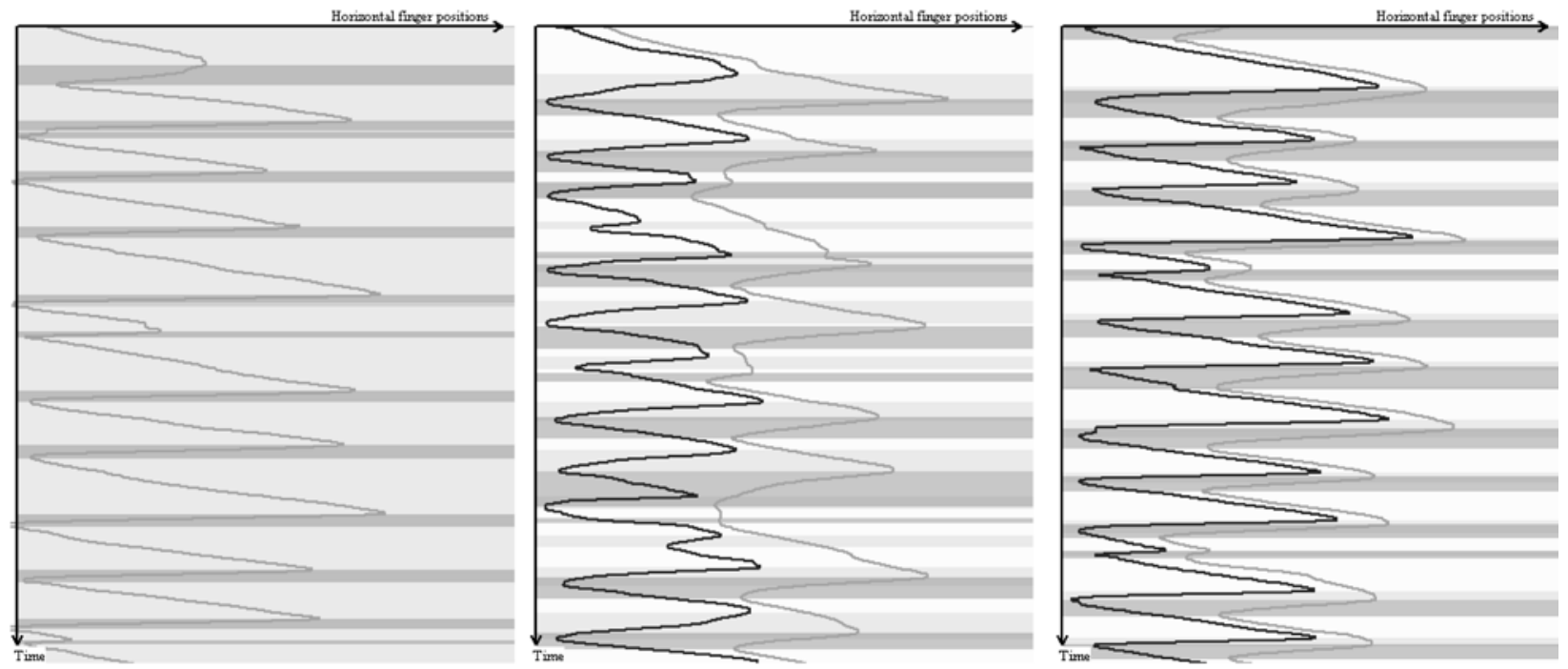

Figure 10. Extended $x-t$ diagram: The visualization in the $x-t$ diagrams has been extended with phase time information by adding horizontal colored stripes corresponding to the four phases: RR, LR, LL, and RL (here shown in gray scale). The reader in A reads only with one hand. The reader in B is a "disjoint" reader, the left part of the sheet is read with the left index finger, and the right part with the right index finger. The reader in $\mathrm{C}$ reads in "mixed mode," there is a substantial area in the middle of the sheet that is read with the two index fingers in parallel.

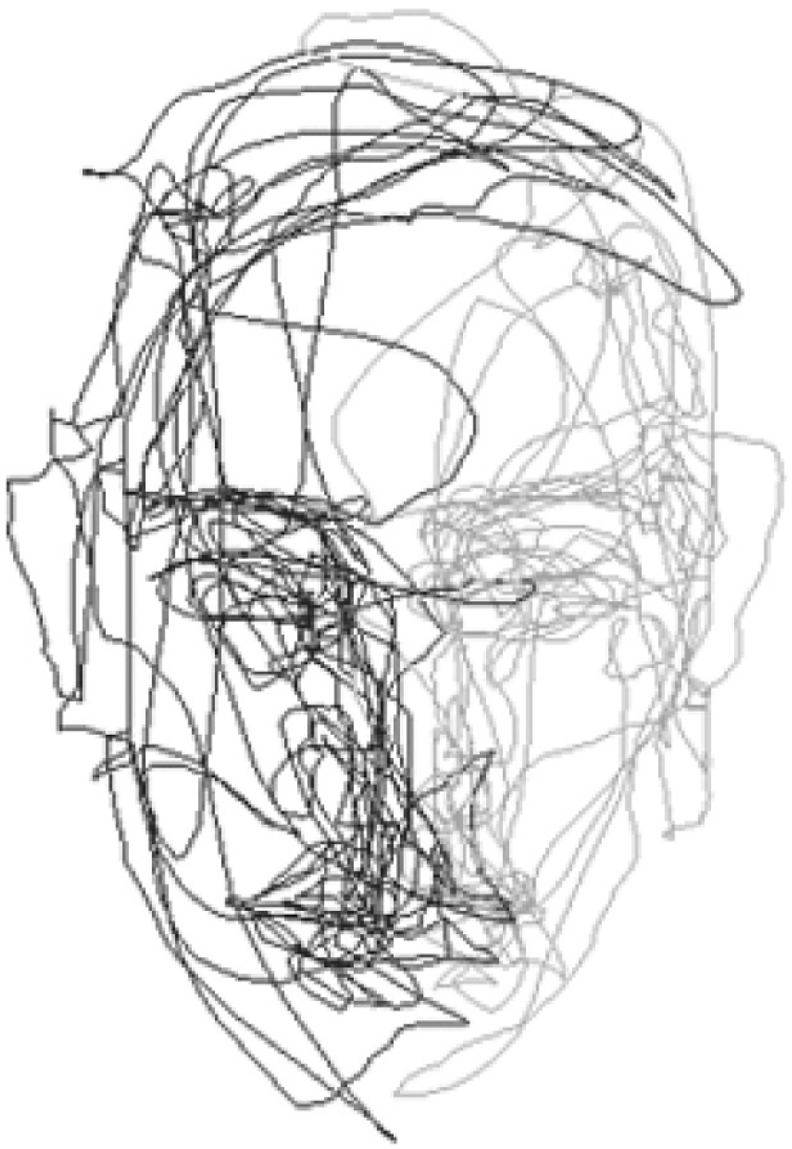

Figure 11. In tactile perception, the two hands can move independently, perhaps simultaneously taking in information from more than one position. The picture shows the trajectories of the left and right index fingers (dark gray and light gray, respectively) during the hands' exploration of a tactile picture of a face. The finger movements cluster around the eyes, mouth, and nose - objects that are criterial to the categorization of the depicted entity as a face. 


$\begin{array}{lrrrr}\text { 'A' } & 0 & 1 & 28 & 16 \\ \text { 'N' } & 0 & 2 & 41 & 16 \\ \text { 'N' } & 0 & 3 & 53 & 16 \\ \text { 'I' } & 0 & 4 & 65 & 16 \\ \text { 'K' } & 0 & 5 & 78 & 16 \\ \text { 'A' } & 0 & 6 & 91 & 16 \\ \text { ' ' } & 0 & 7 & 103 & 16 \\ \text { 'V' } & 0 & 8 & 116 & 16 \\ \text { 'A' } & 0 & 9 & 129 & 16 \\ \text { ॠ } & & & & \\ \text { 'K' } & 0 & 10 & 141 & 16 \\ \text { 'N' } & 0 & 11 & 154 & 16 \\ \text { 'A' } & 0 & 12 & 167 & 16 \\ \text { ' } & & & & \\ \text { 'D' } & 0 & 13 & 179 & 16 \\ \text { 'E' } & 0 & 14 & 192 & 16\end{array}$

Figure 12. Excerpt from an RAI-character-tagged (o) text file for facilitating automatic speech tracking. The name Annika is divided into the three syllables $a n, n i$, and $k a$. The word vaknade (English "woke up") is divided into va, kna, and de. The columns of numbers are for row, column, and $x$-, $y$-coordinates on the Braille sheet.

to also track oral reading on the syllable level, and compare the oral reading position in the text with the finger positions, and how the tactile preread time varied during sentences.

\section{Extendability}

The completely new idea of also tracking the oral reading was not included in the original project aspirations. But the system design tolerated extensions, and most of the basic mechanisms for testing speech tracking were already present in the system.

From the beginning, this was thought to be impossible to implement in the pilot project-a speech recognition system (Becchetti, \& Ricotti, 1999) would have to be used and interfaced to the automatic finger-tracking system. There was doubt about the existence of qualified speech recognition systems that could react on the syllable level, especially in recognizing a minor language such as Swedish.

Instead, an approach based on the interaction between a human analyzer (inherently good at speech recognition and understanding) and a computer program was taken - a semiautomatic way of solving the problem. An easy way to perform speech tracking on a syllable level was to let the program recognize rapid amplitude increases (RAIs) in the oral speech stream. After pauses, RAI events were generated - for example, after pauses between words (not always) and inside words, such as the onset of stop consonants. The word rapid, for example, was divided into the two syllables $r a$ and pid. RAI events were generated not only after complete silent pauses, but anytime the amplitude increases with a certain amount. The human analyzer edited the text file generated from the Braille to the plain text decoder and placed the special RAI event character "o" where he/she judged that an RAI event should occur (within or between words) (see Figure 12). Then the human analyzer ran the automatic finger- and speech-tracking system (see Figure 13). The oral reading was heard from the computer's speakers and a blue frame advanced syllable by syllable on the computer screen. The human analyzer verified if the blue frame sequencing agreed with the oral reading. If there were any disagreements, the human analyzer edited the text file again to add or remove RAI event characters, and then ran the speech-tracking system again. This process was iterated until no errors were found. The human analyzer could run the speech-tracking system at a lower speed, but after a while he or she became so skilled that the playback could be played at full speed. In this first version, it took 15-30 min per Braille sheet to complete the task. With enhanced computer interaction, this time could probably be reduced to

C:Documents and SettingsłbjornLokala inställ... $\square$

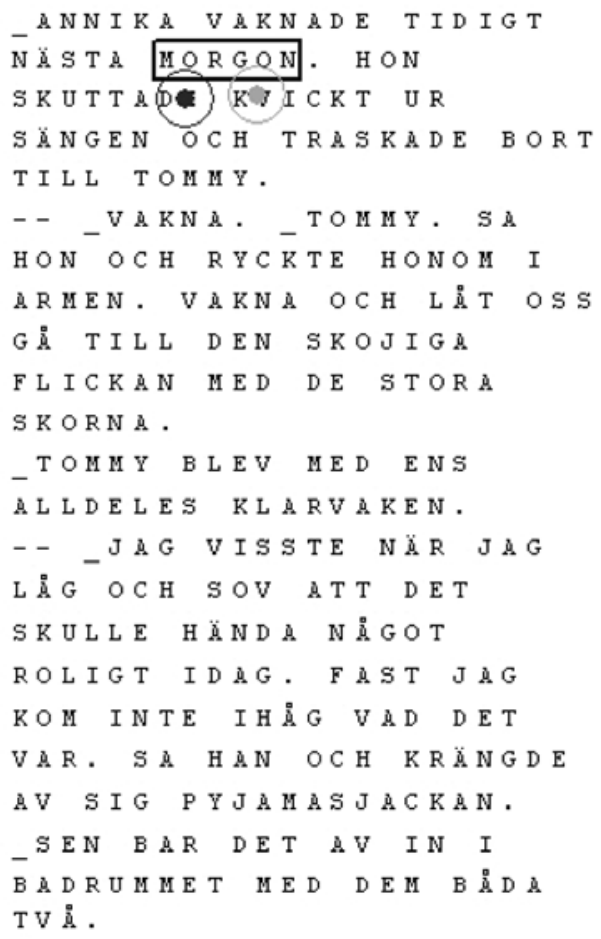

Figure 13. The program extended with speech tracking. The blue rectangle (here in black) follows the reading aloud on the syllable level. 
about $5-10 \mathrm{~min}$. The places for the syllable separating RAI events varied, considerably according to reader, dialect and prosody (e.g., a long vocal could eventually be accentuated by placing a short pause in it, thereby generating an unexpected RAI event after the pause).

Technically, the pause detector was implemented in $\mathrm{C}++$ code in a sample grabber filter in the DirectShow filter graph for the sound path (see Figure 5A). A short segment was taken under consideration. The mean amplitude for this segment was calculated and normalized to the range $[0.0,1.0]$. Then the mean value was compared to the mean value for the previous segment. If the new mean value was larger than the old by a fixed threshold value, the computation generated an RAI event-the amplitude had increased rapidly (determined by the threshold value). Further RAI events were not generated until the mean amplitude had decreased rapidly enough, generating an RAD event (not currently used), and then again increased.

When the syllable-calibration process was completed, and the speech-tracking system was run, a new result file was generated. It contained the positions of the two index fingers and the oral reading for each frame. Those data are

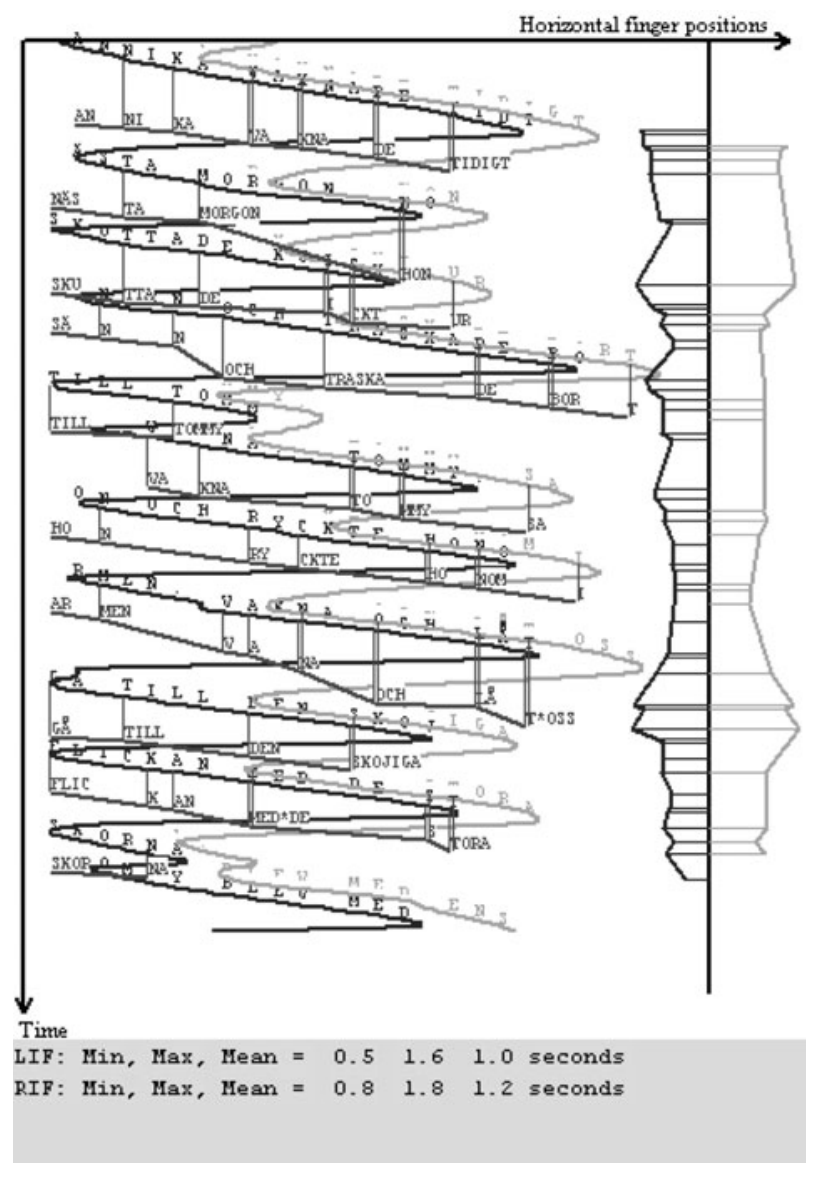

Figure 14. Automatic analysis with both finger and speech tracking (normally in red, green, and blue, but here shown in dark gray, light gray, and middle gray, respectively). The right part of the diagram shows the preread times with respect to the positions of the left and right fingers, and how it varies during reading, especially on the sentence level. visualized in the enhanced $x-t$ diagram (see Figure 14). The red and green traces are visible as before. A blue trace has been added that reflects the timing of the oral reading process. The text accompanies the three traces as they are processed by the fingertips and speech. The right part of each diagram shows the two index fingers' preread time (red and green) with respect to the oral reading. The program has also calculated the minimum, maximum, and mean preread times for left (LIF) and right (RIF) index fingers.

\section{CONCLUSIONS}

The top-down approach and the highly interactive user interfaces, relying on audiovisual feedback, resulted in safe, maintainable, extendable, and use-worthy technology, enabling large scale studies of blind people reading Braille or tactile images. Large scale classifications of different reading styles of many subjects are within reach using the same equipment.

\section{AUTHOR NOTE}

The feasibility study was supported by Swedish Council for Working Life and Social Research (FAS) Grant 2002-0567 and the follow-up project "The Tactile Reading Process" (2006-present). The automatic finger-tracking system is a research-driven technological innovation. It is not a commercial product, and we hope to make it available to broader layers of the research community. Presently, the system only exists in one exemplar, which is located at Lund University. Correspondence concerning this article should be addressed to B. Breidegard, Certec, Division of Rehabilitation Engineering Research, Department of Design Sciences, Lund University, P.O. Box 118, SE-22100 Lund, Sweden (e-mail: bjorn@ certec.lth.se).

\section{REFERENCES}

Becchetti, C., \& Ricotti, L. P. (1999). Speech recognition: Theory and $C++$ implementation. New York: Wiley.

Bertelson, P., Mousty, P., \& D'Alimonte, G. (1985). A study of Braille reading: II. Patterns of hand activity in one-handed and twohanded reading. Quarterly Journal of Experimental Psychology, 37A, 235-256.

Breidegard, B., Eriksson, Y., Fellenius, K., Jönsson, B., HolmQVIST, K., \& STRÖMQVIST, S. (in press). Enlightened: The art of finger reading. Studia Linguistica.

Breidegard, B., Fellenius, K., Jönsson, B., \& StrömQvist, S. (2006). Disclosing the secrets of braille reading: Computer aided registration and interactive analysis. Visual Impairment Research, 3, 49-59.

BROOKNER, E. (1998). Tracking and Kalman filtering made easy. New York: Wiley.

Kruglinski, D., Shepherd, G., \& Wingo, S. (1998). Programming Visual $C++$. Redmond, WA: Microsoft Press.

Linetsky, M. (2001). Programming Microsoft DirectShow. Plano, TX: Wordware Publishing.

Millar, S. (1988). An apparatus for recording hand-movements. British Journal of Visual Impairment \& Blindness, 1, 87-103.

MiLlaR, S. (1997). Reading by touch. London: Routledge.

Mousty, P., \& Bertelson, P. (1985). A study of Braille reading: I. Reading speed as a function of hand usage and context. Quarterly Journal of Experimental Psychology, 37A, 217-233.

Paesce, M. (2003). Programming DirectShow for digital video, television and DVD. Redmond, WA: Microsoft Press.

WirTh, N. (1971). Program development by stepwise refinement. Communications of the ACM (Vol. 14, pp. 221-227). New York: Association for Computing Machinery.

(Manuscript received October 25, 2006; revision accepted for publication February 12, 2007.) 